\title{
Effect of Displacement on Pressure Distribution in Cake Expression
}

\author{
Ndekwu O. B., Ademiluyi J. O. \\ Department of Civil Engineering, Faculty of Engineering, University of Nigeria, Nsukka
}

\begin{abstract}
The principle underlying expression basically involves the separation of a solid from the liquid in which it is suspended by passing the mixture through a porous medium with pore sizes too small to allow the passage of the solid particles at an applied pressure. The analytical method of the finite element method has been used to determine the relationship between pressure distribution and displacement distribution along sludge cake height in a filter press expression process. The finite element formulation basically involves the properties of the sludge in question as distinguished from other solution. The solution domain was idealized as a one-dimensional quadratic shape function for the purpose of this analysis and the displacement function formulation method were employed in solving the pressure distribution. It was discovered that pressure increases with an increase in displacement of the cake at different time of expression. It was also discovered that pressure increases simultaneously with displacement as the time of expression increases. The ranges of displacement in a sludge cake height with increase time of expression increases with a decrease in sludge depth, hence impacting positively of the effective pressure. The displacement increases continuously with increase time of expression.
\end{abstract}

Keywords- Expression, Sludge cake, Compressive pressure.

\section{INTRODUCTION}

Cake filtration is an important and widely used separation process in many areas in which the objectives may be to either recover a clear filtrate, or to recover the suspended solids, or both. The aforementioned areas include the chemical and petrochemical, mineral, food, pharmaceutical and water treatment industries. In the process industries, separation and purification of the product stream is probably the stage where most value is added. Also, increasing environmental awareness has resulted in ever more stringent legislations to control liquid discharge compositions. These two factors have led to separation science and technology playing a pivotal role in the relationship between product quality, competitiveness and the environment (Tien, 2002).

The handling and disposal of this sludge is one of the greatest challenges facing the environmental engineer. The sludge has high water content and compressibility attribute and as such it is expedient to dewater it to reduce its volume and prevent environmental health hazard. The dewatering of sludge using the filter press method has been in existence far back as in the 1920s. Since then, a number of equations have been presented by various contributors aimed at improving the performance of the sludge filtration/expression process, Carman, (1934), (1938); Grace, (1953); Coackley, (1956); Ademiluyi and others, (1983), (1987); Gale, (1975); Anazodo, (1974); Ademiluyi, (1986). However, their research was limited to experimental work which could not provide an insight into the interactive nature of sludge filterability. As previously sighted in literature (Anazodo, 1974; Ademiluyi, 1986) the inapplicability of Darcy's law of fluid flow to the dewatering process stems from the fact that Darcy's law is only applicable to rigid materials where porosity is constant. This uniformity in porosity implies a corresponding uniformity in pressure throughout the cake height. This is not true with compressible or deformable materials in for sludge cake. Porosity decreases from sludge to the cake height closest to the septum (Hemant, 1981; Bear et al, 1991; Lee et al, 2000; Svarosky, 2000; Challeppah et al, 2009; Challeppah et al, 2010). In the course of this porosity variation, pressure also varies as a result of solid compaction displacement along the sludge height. Even though the direction of porosity and pressure variation will be intuitively assessed, there is still the need to provide a theoretical base for such assessment. It is in the light of the foregoing that finite element method of analysis was used in this study to evaluate the effect of displacement distribution on effective pressure along the expressed cake height. It is hoped that the study will find practical application in the evaluation of models necessary to describe cake expression phenomenon. 


\section{RESULTS AND DISCUSSION}

As presented in previous publications, the finite element model equation for pressure distribution using a displacement formulation matrix has been developed. The quadratic shape function model was used on a cake expression generated data and the results are as presented below.
A graphical representation of pressure and displacement at particular heights of sludge cake versus time as shown in Figures (1, 2, 3 and 4) indicates that pressure increases with time. This is attributed to the continuous dissipation of excess pore water pressure with increased filtration time.

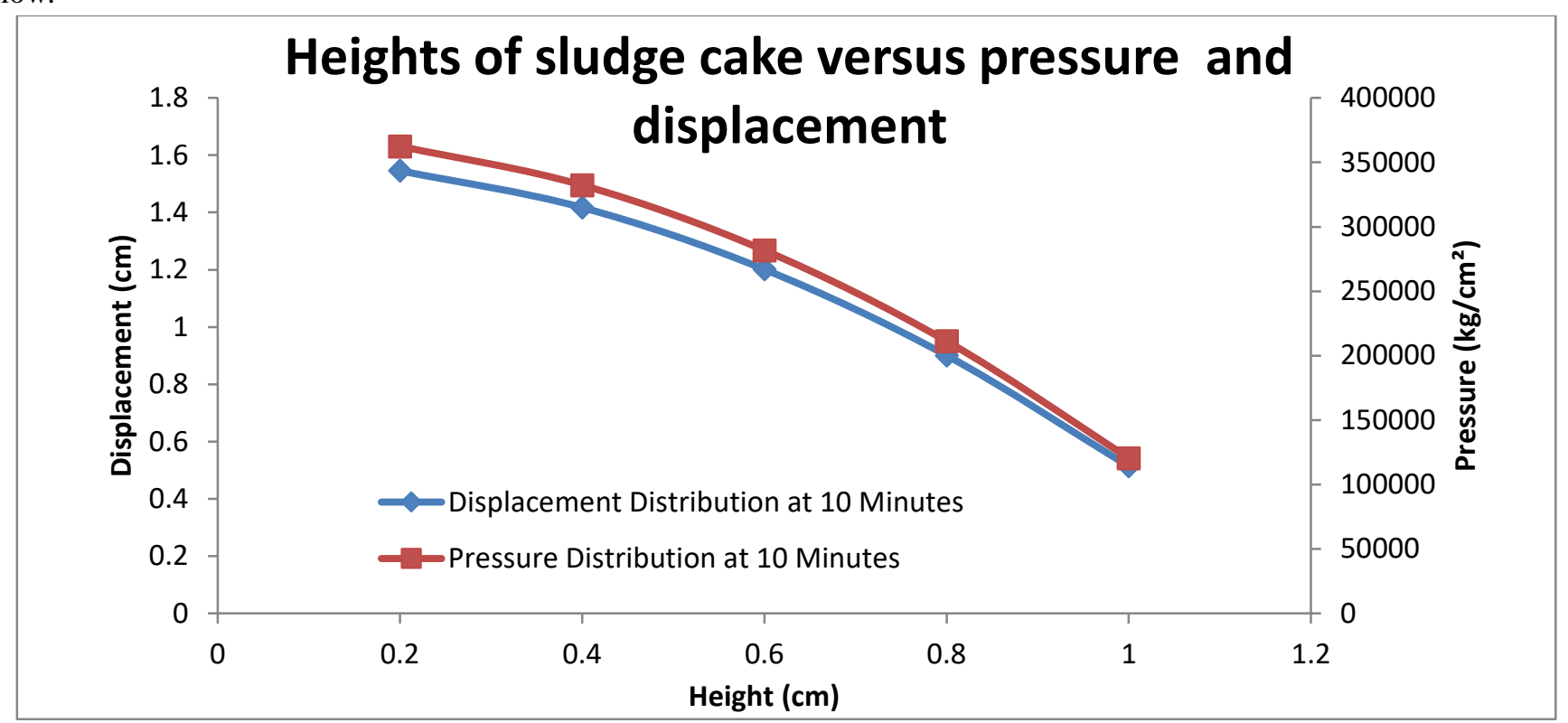

Fig.1: Plot of Heights of sludge cake versus pressure and displacement at 10 minutes

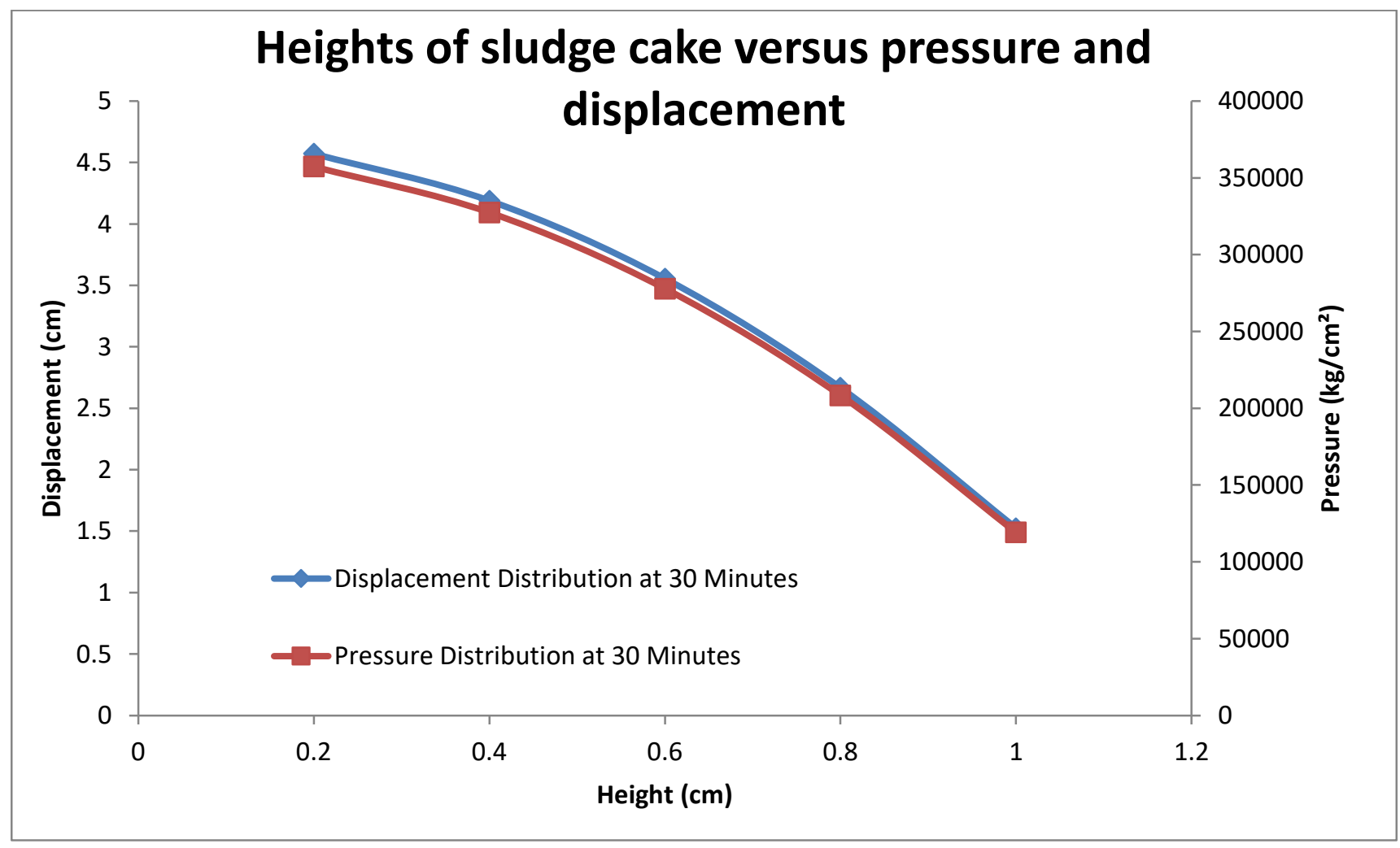

Fig.2: Plot of Heights of sludge cake versus pressure and displacement at 30 minutes 
It is evident, that from the plot of height of sludge versus pressure and displacement, there is a gradual trend to uniformity of pressure and displacement along the cake height as the time of expression increases. Hence the rate of displacement is directly proportional to the compressive pressure, which implies that the internal pressures are functions of both position and time. The effective pressure is not a physical quantity. The effective pressure is then simply the drag on all the particles in the distance from $\mathrm{x}$ to $\mathrm{L}$ divided by the cross-sectional area. This implies that the drop in hydraulic pressure from water pores across the sludge length resulting in an increase in displacement is exactly equal to the rise in solid compressive pressure.

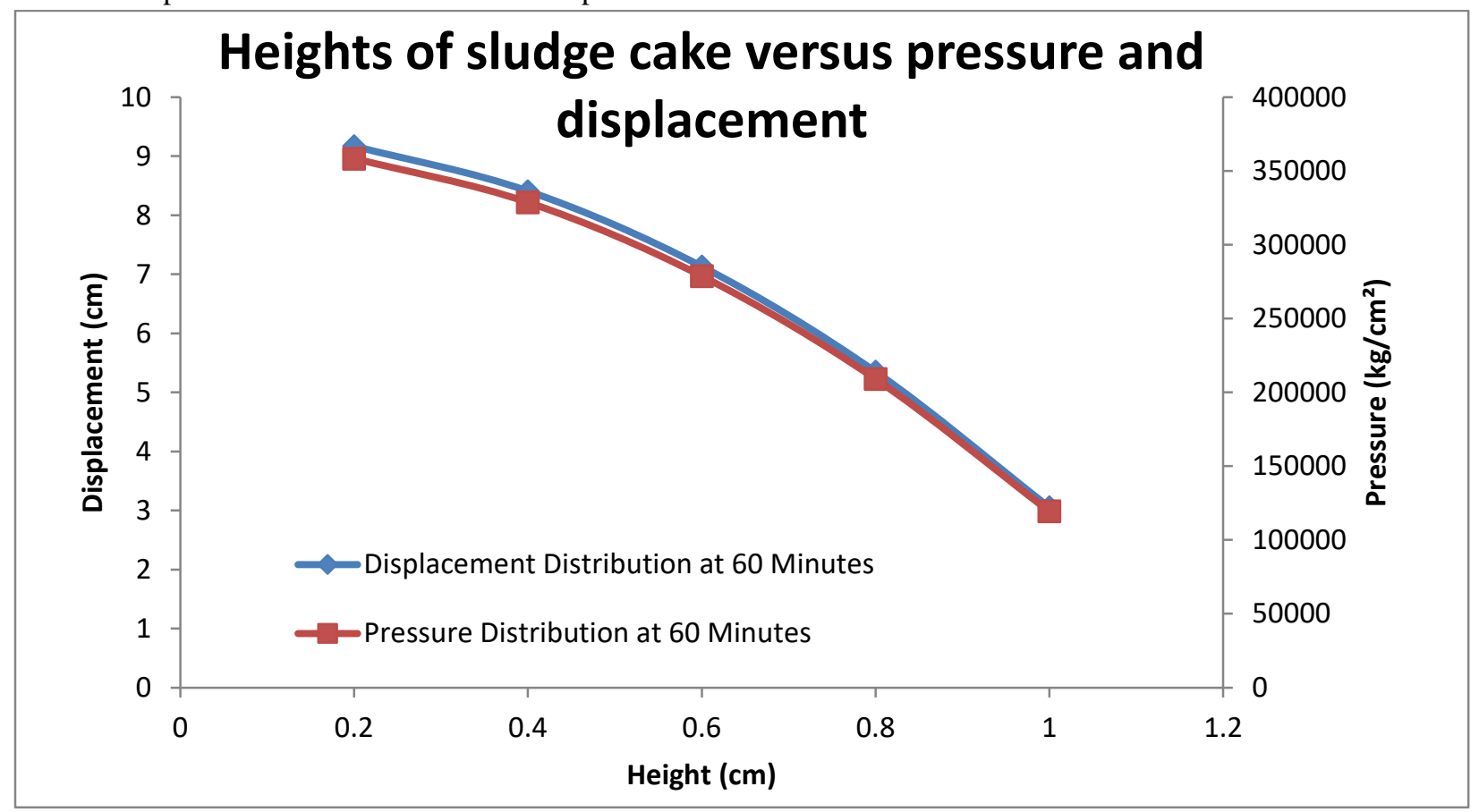

Fig.3: Plot of Heights of sludge cake versus pressure and displacement at 60 minute

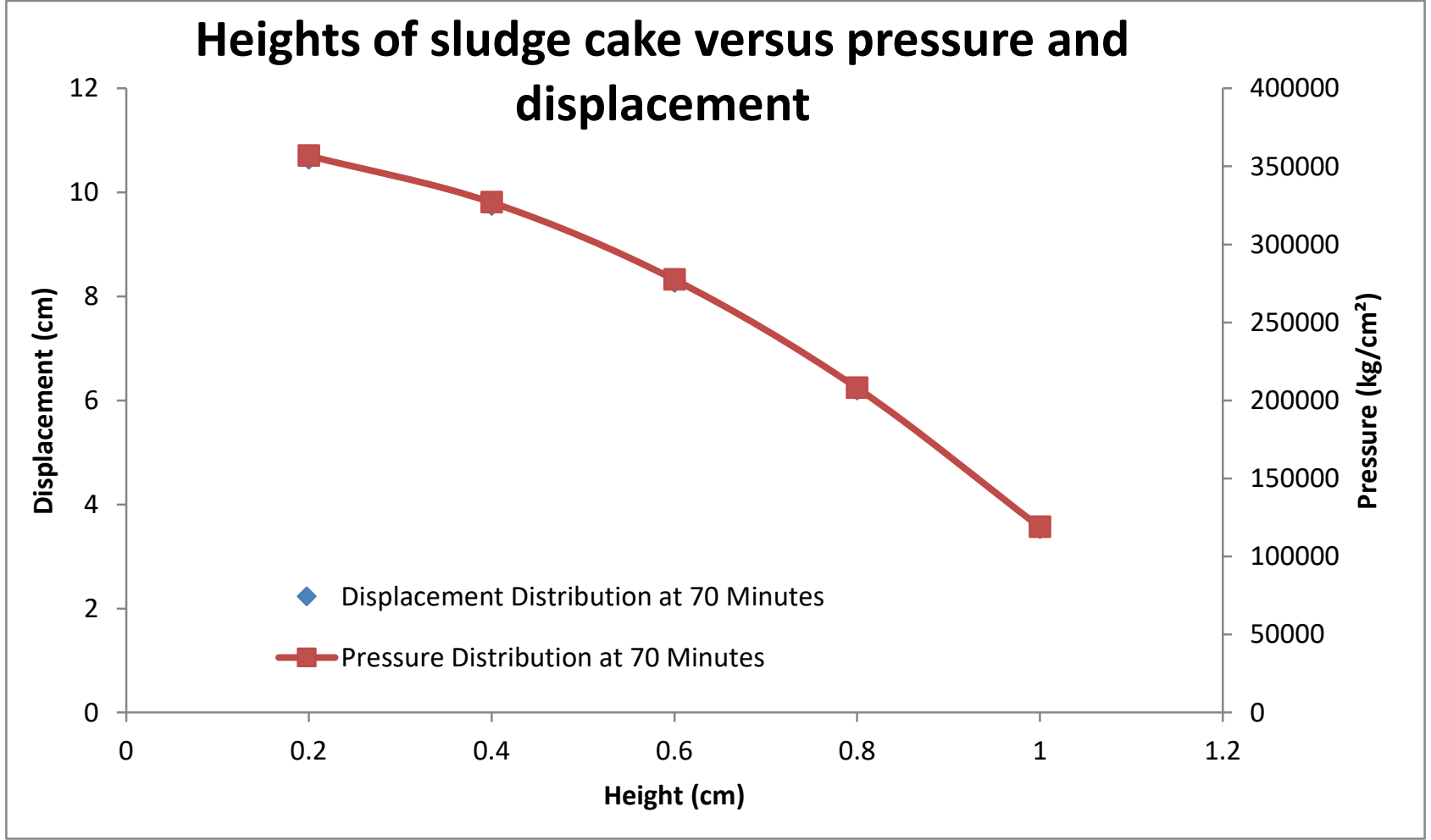

Fig.4: Plot of Heights of sludge cake versus pressure and displacement at 70 minutes 
Sludge filterability is a function of porosity which in turn depends on the pressure difference across the filter cake. Therefore the study of pressure distribution along height of sludge cake can aid in the variation of the filterability parameter used to quantify sludge dewatering. A decrease in the depth of the expressed cake, results in a decrease in the pressure drop. Hence, an increase in displacement implies a corresponding increase in pressure change. It is important to note that the effective pressure is directly proportional to the compressive pressure whose values especially in filter presses could be negative indicating the fact that it is a counteractive force (compressive), which when compared with the axial strain deformation, as shown in Figures $(5,6)$ displays a correlative result (i.e. the higher the axial strain deformation, the greater the effective pressure distribution).

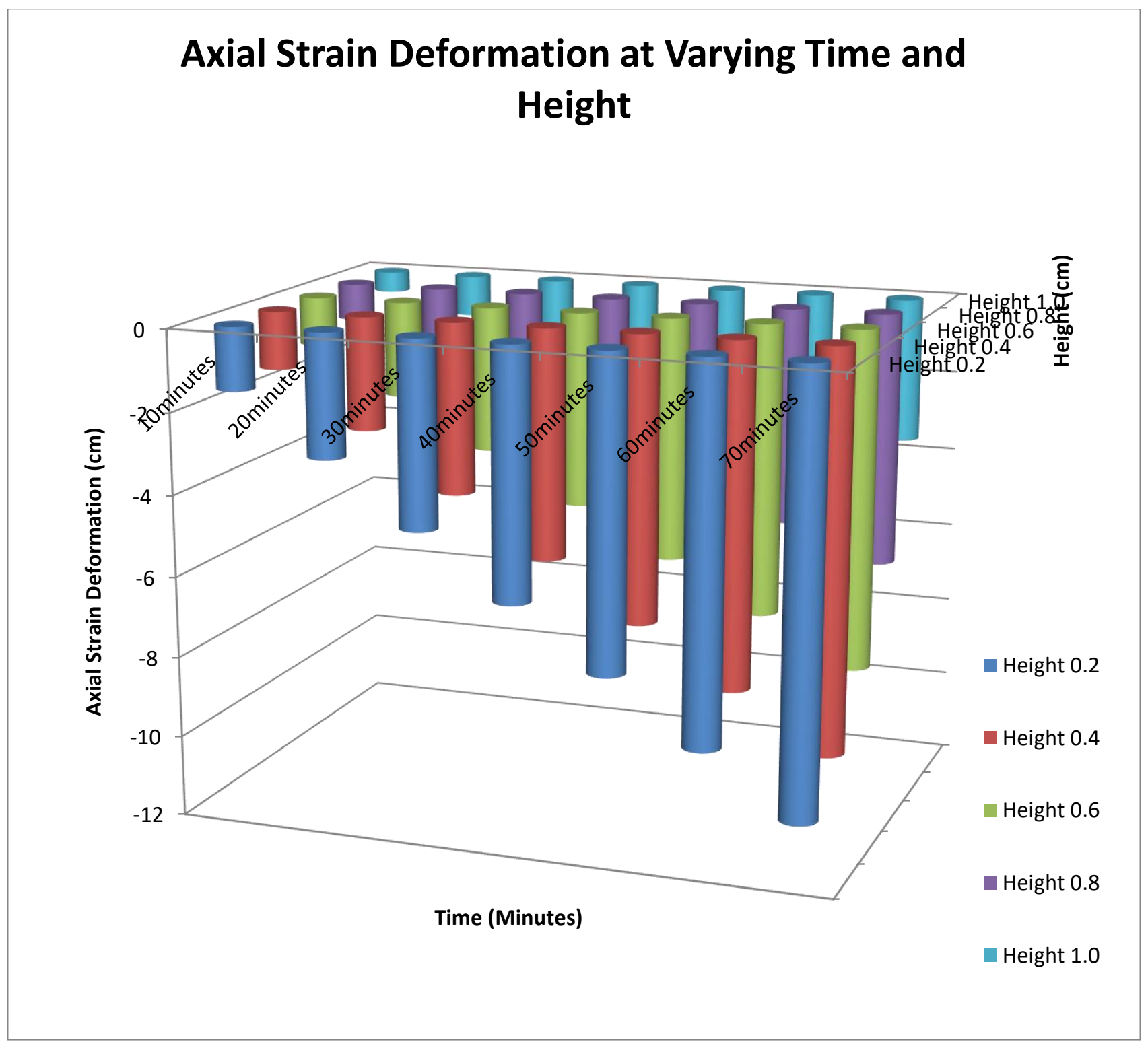

Fig.5: Plot of Axial Strain Deformation at Varying Time and Height 


\section{Effective Stress at Varying Time and Height}

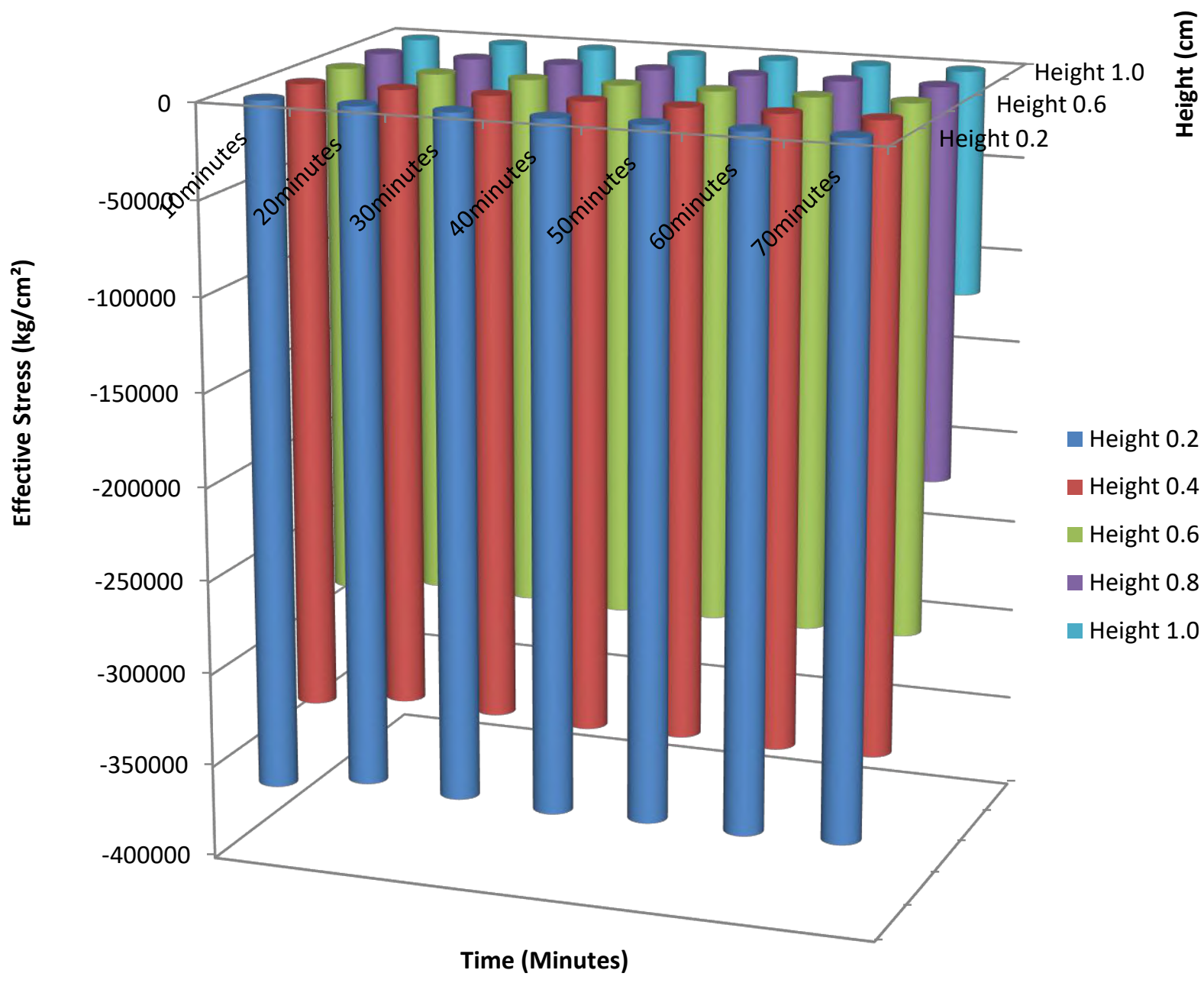

Fig.6: Plot of Effective Stress Distribution at Varying Time and Height

At the beginning of the expression the whole pressure drop available is across the medium itself since as yet no cake is formed. As the pores of the medium are normally small and the rate of filtrate flow is slow, the flow conditions are almost invariably laminar (Tiller, 1974, Svarovsky, 2000). When the particles are retained at the surface of the medium, they form a cake. As soon as the first layer of the cake is formed, the subsequent expression takes place on the top of this cake and the medium provides only a supporting function. A greater proportion of the available pressure drop is taken up by the cake itself. This results in an effective increase in the bed resistance as a result of solid particle compaction (displacement) thus leading to a gradual drop in the filtrate flow rate in constant pressure expression.

\section{CONCLUSION}

1) It has been demonstrated that the expression mechanisms under constant pressure consist of two flow phenomenon; of filtration and consolidation, and these phenomena, though occurring progressively from filtration, changes at a transition stage with increased time of filtration, with no visible flow of filtrate, but with evidences of displacement in the sludge cake height as a result of solid settlement. A process called consolidation. 
2) The rate of displacement is directly proportional to the compressive pressure, which implies that the internal pressures are functions of both position and time.

3) The drop in hydraulic pressure from water pores across the sludge length results in an increase in displacement which is exactly equal to the rise in solid compressive pressure.

\section{REFERENCES}

[1] Ademiluyi J. O. (1986). Development in the constant Vacuum cake filtration theory: Proceedings of the Engineering section of science association of Nigeria Vol. 6 \& 7.

[2] Ademiluyi, J. O. Egbuniwe, N. and Agunwamba, J. C. (1987). A dimensionless number as an index of sludge dewaterability, Journal of Engineering for Development, Vol. 1, p. 1-12.

[3] Ademiluyi, J.O. Anazodo, U.G. and Egbuniwe, N. (1983). Filterability and compressibility of sludge pt 11. Effluent and water treatment journal vol. 23, no 1 pp 25-30.

[4] Anazodo U.G. (1974). Dimensional equation for sludge filtration, effluent and water treatment journal vol, 14 n0 9 pp517-523.

[5] Bear, J. and Bachmat, Y. (1991), Introduction to Modeling of Transport Phenomena in Porous Media, Kluwer Academic Publishers.

[6] Carman, P.C. (1934). A Study of Mechanism of Filtration Part II, Journal of the Society of Chemical Industry, Transactions Communication. 53(6):15911651.

[7] Carman, P.C. (1938). Fundamental Principles of Industrial Filtration. Transactions - Institution of Chemical Engineers; 16: 168-188.

[8] Chellappah K. Tarleton E.S. and Wakeman R.J. (2009). Cake filtration and sedimentation of fibre/particle mixtures, Poster session presented at: 10th UK Particle Technology Forum, 2009 Jul 1-2; Birmingham, UK (won 2nd place in poster competition).

[9] Chellappah K. Tarleton E.S. and Wakeman R.J. (2010). The porosity, permeability and restructuring of heterogeneous filter cakes, Chemical Engineering and Technology (In Press).

[10] Coackley, P. and Jones, R.S. (1956). Vacuum sludge filtration, Sewage and industrial waste journal.

[11] Gale, R.S. (1975). Comment on dimensional equation, Effluent and water treatment journal, p 422.

[12] Grace, H.P. (1953). Resistance and Compressibility of Filter Cakes, Part II: Under Conditions of Pressure
Filtration, Chemical Engineering Progress, Vol. 49, No. 7, pp. 367-377.

[13] Hemant, R. M. (1981). Cakes filtration empirically incorporating parcile migration, Filtration and separation. Vol 18, No. 1,pp. 20-24.

[14]Lee, D. J. and Wang, C. H. (2000). Theories of cake filtration and consolidation and implications to sludge dewatering, Water Research, vol. 34(1), pp. 1-20

[15] Svarovsky, L.( 2000). Solid-Liquid Separation, 4th Ed., Butterworth-Heinemann, Oxford, UK, pp. 1-29, 303-334, 335-348, 368-392.

[16] Tien, C. (2002). Cake filtration research- A personal view, Powder Technology 127, 1-8.

[17] Tiller, F.M. (1974). What the Filter Man Should Know about Filtration, re-published in Filtration (Special Issue), 2004, pp. 55-67. 